\title{
Traumatic subarachnoid hemorrhage, basal ganglia hematoma and ischemic stroke caused by a torn lenticulostriate artery
}

\author{
Christian Fung • Werner J. Z'Graggen • Jürgen Beck • \\ Jan Gralla $\cdot$ Stephan M. Jakob $\cdot$ Philippe Schucht • \\ Andreas Raabe
}

Received: 8 June 2011 / Accepted: 2 September 2011 /Published online: 6 October 2011

(C) Springer-Verlag 2011

\begin{abstract}
Subarachnoid hemorrhage (SAH), basal ganglia hematoma (BGH) and ischemic stroke are common diseases with diverging therapies. The simultaneous occurrence of these diseases is rare and complicates the therapy. We report the case of a 30-year-old man with a ruptured lenticulostriate artery after traumatic brain injury that caused the combination of SAH, BGH and ischemic stroke and subsequent cerebral vasospasm. This rupture mimicked the pathophysiology and imaging appearance of aneurysmal SAH. The site of rupture was not secured by any treatment; however, hyperdynamic therapy and percutaneous transluminal angioplasty were feasible in this setting to prevent additional delayed neurological deficit.
\end{abstract}

Sources of funding Financial support for patient care and treatment was provided by Bern University Hospital, Department of

Neurosurgery, Bern, Switzerland. No additional funding was received.

This work has not been previously published or presented.

C. Fung · W. J. Z'Graggen · J. Beck · P. Schucht · A. Raabe $(\bowtie)$

Department of Neurosurgery,

Bern University Hospital and University of Bern,

c/o Publication Office, Susan Wieting,

3010 Bern, Switzerland

e-mail: susan.wieting@insel.ch

J. Gralla

Institute of Neuroradiology, Bern University Hospital and

University of Bern,

3010 Bern, Switzerland

S. M. Jakob

Department of Intensive Care Medicine,

Bern University Hospital and University of Bern,

3010 Bern, Switzerland

\section{Introduction}

Subarachnoid hemorrhage (SAH) is a common finding in traumatic brain injury (TBI) [5]. The incidence of basal ganglia hematoma (BGH) based on shearing of the lenticulostriate arteries however is not a common entity $[1,8,19]$ nor is there a wide body of literature regarding focal ischemic lesions after TBI $[4,9,10]$. Each disease has distinct and partially diverging therapy regimes, and the simultaneous occurrence of all three entities complicates the clinical course [20]. Therapy for deep-seated hematomas is mainly conservative and includes the management of arterial blood pressure to prevent recurrent hemorrhage, intracranial pressure and other medical complications [2]. Treatment of ischemic stroke varies and depends on the etiology [6]. In SAH, the two major causes are either trauma or aneurysmal bleeding. Traumatic subarachnoid hemorrhage (tSAH) shows distinct differences when compared to aneurysmal subarachnoid hemorrhage (aSAH) [7]. With tSAH, onset time and severity of vasospasm are generally earlier and of milder course, respectively [13, 21]. In addition, tSAH is often located toward the convexity of the hemisphere. However, if tSAH originates from large vessels, radiological findings as well as the clinical course might mimic aneurysmal hemorrhage with severe vasospasm and the need for neuro-intervention $[3,11,12,16$, 17, 20, 21]. To our knowledge this is the first report describing the occurrence of $\mathrm{SAH}, \mathrm{BGH}$ and ischemic stroke secondary to TBI. The aim of this report is to describe the combination of the lesions, to highlight the probability of severe cerebral vasospasm with severe tSAH and the feasibility of angioplasty in the setting of a ruptured lenticulostriate artery. 


\section{Case report}

A male patient 30 years of age was admitted after getting into a brawl that resulted in an impact to the right side of his head. Upon neurological examination, the patient was disoriented to time and place, with eye opening to speech and a left-sided hemiparesis [analogous to Glasgow coma scale (GCS) score of 13]. A CT scan showed a SAH analogous to aSAH Fisher grade 3 [7], an intracerebral hematoma within the cerebral peduncle, crus posterius of the internal capsule and the medial thalamus on the right, and a fracture of the skull base (Fig. 1a, b).

The patient was intubated because of a drop in GCS score and severe agitation. Assuming that a ruptured aneurysm or an artery dissection caused the SAH, cerebral angiography was performed on the day of admission, but yielded no pathological results. Because of the Fisher grade 3 bleeding, treatment with nimodipine was started. The next day the patient was extubated, still agitated and had a leftsided hemiplegia. A CT scan $20 \mathrm{~h}$ after admission showed, in addition to the initial findings, ischemic basal ganglia infarction on the right side. Magnetic resonance imaging (MRI) $14 \mathrm{~h}$ later confirmed sub-acute ischemic infarction but showed no signs of axonal shearing injuries on T2weighted image or susceptibility weighted imaging (SWI) despite moderate head trauma (Fig. 1b-d). In the following days, CT scan ruled out hydrocephalus. However, transcranial Doppler (TCD) sonography showed a mild increase of mean velocities from 780 to $1,340 \mathrm{~mm} / \mathrm{s}$ (days 2 to 4 ) of the left middle cerebral artery (MCA). The patient was clinically stable, oriented to person and place with a persisting left-sided hemiplegia [GCS 14, NIH Stroke Scale (NIHSS) 12].

Five days after the trauma, the GCS score dropped to 12, and the NIHSS score increased to 16. In addition, there was an increase of mean TCD velocities of the right MCA from 930 to $3,330 \mathrm{~mm} / \mathrm{s}$ (days 4 to 5 ). Cerebral angiography was performed and confirmed severe vasospasm of the intracranial segments of both internal carotid arteries (ICA) and the right MCA. Percutaneous transluminal angioplasty
Fig. 1 CT scan at admission revealed typical severe $\mathrm{SAH}$ predominately in the basal cistern and Sylvian fissure (a) following severe head trauma. MRI revealed a hemorrhagic lesion in the caudal aspect of the right basal ganglia (SWI, b) and an ischemic lesion of the right basal ganglia in

diffusion-weighted imaging (c) and apparent diffusion coefficient maps (d)
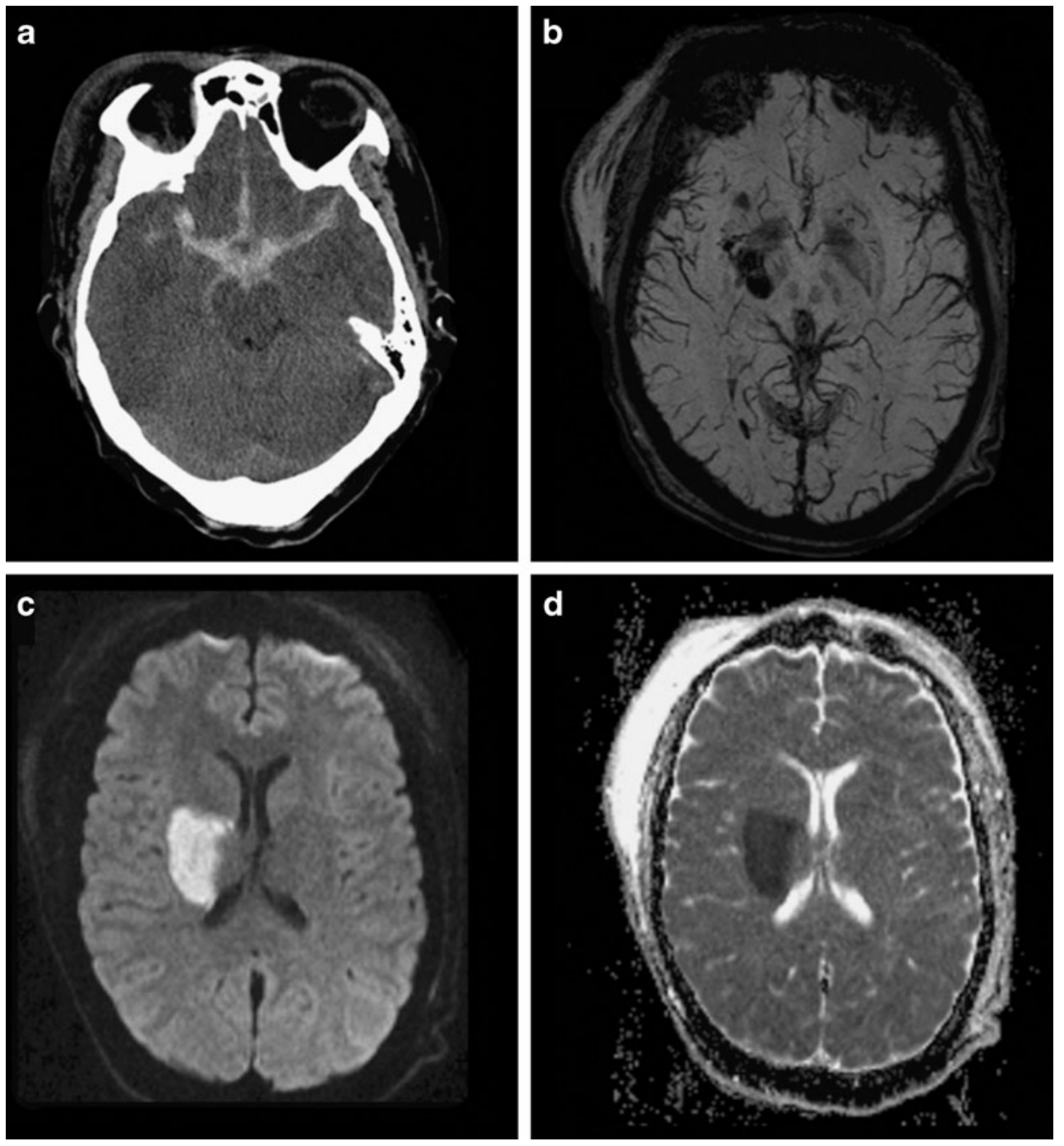
(PTA) for the ICA on both sides and MCA on the right side were performed (Fig. 2). Using hyperdynamic therapy with a mean arterial pressure of $100 \mathrm{mmHg}$, the clinical course stabilized [15]; however, on day 8, the patient presented with a new onset of mild hemiparesis on the right side and a mean velocity of $2,380 \mathrm{~mm} / \mathrm{s}$ of the left MCA. Cerebral angiography was performed and again confirmed severe vasospasm of the left MCA and the left distal ICA. Both locations of vasospasm were treated by PTA. Right-sided neurological deficits resolved completely after PTA, and TCD velocity dropped significantly $(1,400 \mathrm{~mm} / \mathrm{s})$. The patient was alert and neurologically stable with a GCS score of 14 and NIHSS score of 12 for 2 days. However, on day 10 he had a relapse of right-sided hemiparesis (NIHSS 16) and increasing TCD velocity $(2,430 \mathrm{~mm} / \mathrm{s})$ of the left MCA. Another diagnostic angiography, followed by PTA of both ICAs and the left MCA, was necessary in order to restore sufficient perfusion.

The patient improved again to the initial GCS and NIHSS scores with no clinical evidence for vasospasms during the following days. Hyperdynamic therapy stopped on day 14. On discharge (day 15) the patient presented with left-sided hemiplegia but no other deficit. The highest mean TCD velocity at discharge was $830 \mathrm{~mm} / \mathrm{s}$ at the right anterior cerebral artery (ACA).

\section{Discussion}

The combination of the various lesions consisting of SAH, $\mathrm{BGH}$ and ischemia suggests a rupture of a lenticulostriate artery. Other vascular causes for SAH and ischemia, such as aneurysmal bleeding or traumatic artery dissection, could not be detected with MRI and cerebral angiography. Direct trauma-associated bleeding in the course of the skull base fracture seemed unlikely to us since we would have expected at least some blood in the epidural or subdural space if the fracture had been responsible for the SAH analogous to Fisher grade 3 . To our knowledge, this is the first report of a ruptured lenticulostriate artery that caused clinical and CT scan typical aSAH, ischemic infarction of the basal ganglia and BGH as a result of head trauma. Because of the typical presence of subarachnoid and parenchymal hemorrhage, we assumed that the rupture of the artery occurred at, or near, the branching site from the M1 segment of the MCA and that the disruption of blood supply caused the circumscribed ischemia in the basal ganglia.

Treatment of this patient was intended to prevent delayed neurological deficit as a consequence of cerebral vasospasm. The highest risk for developing hemodynamically significant vasospasm after tSAH occurs within the first 7-8 days after trauma [13], whereas vasospasm after aSAH can occur up to 14 days after aneurysm rupture [18]. Additionally, mean TCD velocities are reported to be lower after tSAH compared to aSAH [13]. In this case, however, the highest mean TCD velocity was at day 5 , and at day 10 clinical evidence and angiographically proven vasospasm were present.

Radiological and TCD findings, as well as the need for three treatments with PTA up to 10 days after trauma, resemble the characteristics of cerebral vasospasm after aSAH rather than those reported after tSAH. Although the site of rupture was not secured, aggressive treatment of the vasospasm was indicated in order to preserve right-sided motor function and prevent imminent infarction. We decided to proceed with induced hypertension as well as endovascular angioplasty based on the results of treatment of severe vasospasm in the presence of unsecured aneurysms [14]. Neither PTA nor hyperdynamic therapy caused rebleeding.
Fig. 2 The digital subtraction angiography of the right ICA territory shows severe vasospasm of the MCA and ACA (a). After local administration of $2.5 \mathrm{mg}$ nimodipine into the terminal ICA and balloon dilatation of the ICA and M1 segment of the MCA distal ICA and proximal MCA increased in diameter (b)
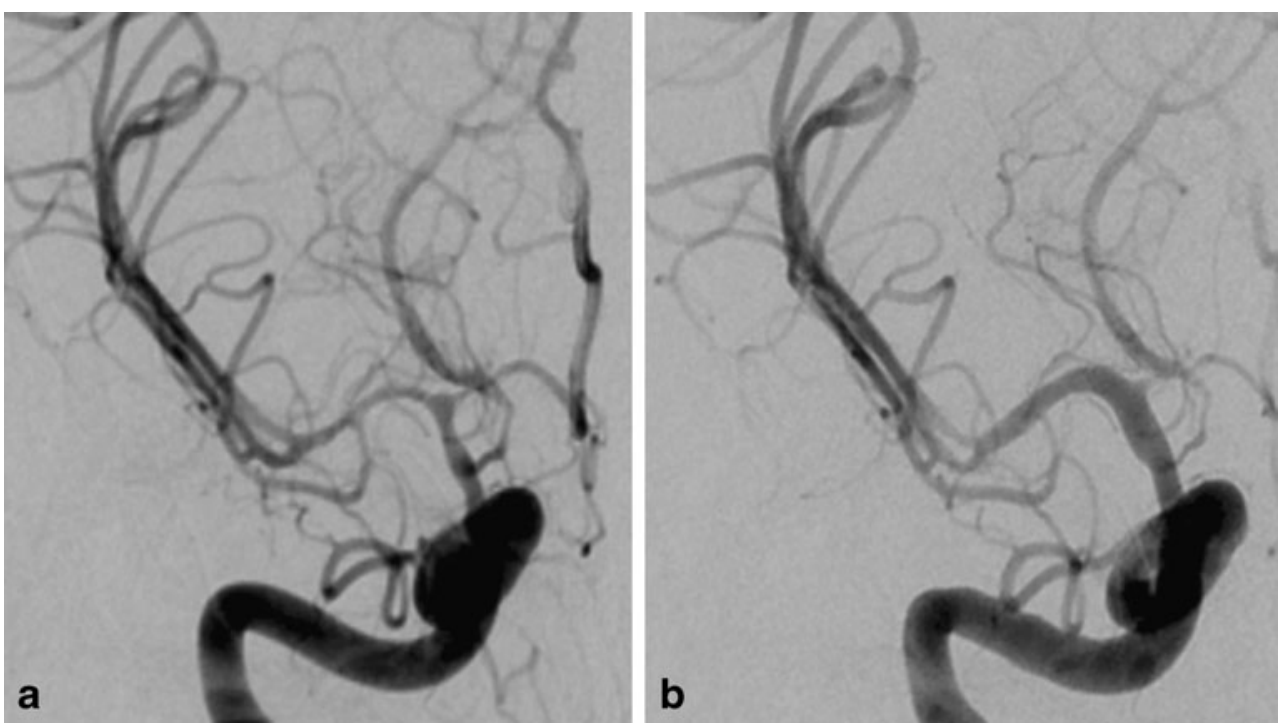


\section{Conclusions}

This case highlights (1) that a ruptured artery might mimic the pathophysiology of aSAH with the risk of cerebral vasospasm, (2) the necessity of neurointensive care to monitor and treat possible delayed neurological deficits after tSAH comparable to Fisher grade 3 and (3) that treatment of cerebral vasospasm such as hyperdynamic therapy and PTA seems to be feasible in the setting of a ruptured unsecured artery.

Acknowledgments The authors received editing support for the final manuscript by Susan Wieting, Bern University Hospital, Department of Neurosurgery, Publication Office, Bern, Switzerland.

Conflicts of interest None.

\section{References}

1. Boto GR, Lobato RD, Rivas JJ, Gomez PA, de la Lama A, Lagares A (2001) Basal ganglia hematomas in severely head injured patients: clinicoradiological analysis of 37 cases. J Neurosurg 94:224-232

2. Broderick J, Connolly S, Feldmann E, Hanley D, Kase C, Krieger D, Mayberg M, Morgenstern L, Ogilvy CS, Vespa P, Zuccarello M (2007) Guidelines for the management of spontaneous intracerebral hemorrhage in adults: 2007 update: a guideline from the American Heart Association/American Stroke Association Stroke Council, High Blood Pressure Research Council, and the Quality of Care and Outcomes in Research Interdisciplinary Working Group. Circulation 116:e391-e413

3. Conti A, Angileri FF, Longo M, Pitrone A, Granata F, La Rosa G (2008) Intra-arterial nimodipine to treat symptomatic cerebral vasospasm following traumatic subarachnoid haemorrhage. Technical case report. Acta Neurochir (Wien) 150:11971202

4. Dharker SR, Mittal RS, Bhargava N (1993) Ischemic lesions in basal ganglia in children after minor head injury. Neurosurgery 33:863-865

5. Eisenberg HM, Gary HE Jr, Aldrich EF, Saydjari C, Turner B, Foulkes MA, Jane JA, Marmarou A, Marshall LF, Young HF (1990) Initial CT findings in 753 patients with severe head injury. A report from the NIH Traumatic Coma Data Bank. J Neurosurg 73:688-698

6. European Stroke Organisation (2008) Guidelines for management of ischaemic stroke and transient ischaemic attack. Cerebrovasc Dis 25:457-507
7. Fisher CM, Kistler JP, Davis JM (1980) Relation of cerebral vasospasm to subarachnoid hemorrhage visualized by computerized tomographic scanning. Neurosurgery 6:1-9

8. Katz DI, Alexander MP, Seliger GM, Bellas DN (1989) Traumatic basal ganglia hemorrhage: clinicopathologic features and outcome. Neurology 39:897-904

9. Kieslich M, Fiedler A, Heller C, Kreuz W, Jacobi G (2002) Minor head injury as cause and co-factor in the aetiology of stroke in childhood: a report of eight cases. J Neurol Neurosurg Psychiatry 73:13-16

10. Kuroiwa T, Tanabe H, Takatsuka H, Arai M, Ozaki T, Nagasawa S, Ohta T (1994) Paramedian thalamic infarction following blunt head injury-case report. Neurol Med Chir (Tokyo) 34:20-22

11. Lee DJ, Moftakhar P, Glenn TC, Vespa PM, Martin NA (2008) Intra-arterial calcium channel blocker infusion for treatment of severe vasospasm in traumatic brain injury: case report. Neurosurgery 63:E1004-E1006

12. Martin NA, Doberstein C, Alexander M, Khanna R, Benalcazar H, Alsina G, Zane C, McBride D, Kelly D, Hovda D et al (1995) Posttraumatic cerebral arterial spasm. J Neurotrauma 12:897-901

13. Oertel M, Boscardin WJ, Obrist WD, Glenn TC, McArthur DL, Gravori T, Lee JH, Martin NA (2005) Posttraumatic vasospasm: the epidemiology, severity, and time course of an underestimated phenomenon: a prospective study performed in 299 patients. J Neurosurg 103:812-824

14. Platz J, Güresir E, Vatter H, Berkefeld J, Seifert V, Raabe A, Beck $\mathrm{J}$ (2011) Unsecured intracranial aneurysms and induced hypertension in cerebral vasospasm: is induced hypertension safe? Neurocrit Care 14:168-175

15. Raabe A, Beck J, Keller M, Vatter H, Zimmermann M, Seifert V (2005) Relative importance of hypertension compared with hypervolemia for increasing cerebral oxygenation in patients with cerebral vasospasm after subarachnoid hemorrhage. J Neurosurg 103:974-981

16. Shahlaie K, Boggan JE, Latchaw RE, Ji C, Muizelaar JP (2009) Posttraumatic vasospasm detected by continuous brain tissue oxygen monitoring: treatment with intraarterial verapamil and balloon angioplasty. Neurocrit Care 10:61-69

17. Taneda M, Kataoka K, Akai F, Asai T, Sakata I (1996) Traumatic subarachnoid hemorrhage as a predictable indicator of delayed ischemic symptoms. J Neurosurg 84:762-768

18. Weir B, Grace M, Hansen J, Rothberg C (1978) Time course of vasospasm in man. J Neurosurg 48:173-178

19. Yamakawa N, Furuno M, Okada M, Waga S (1995) Traumatic basal ganglia haemorrhage: report of 7 cases. J Clin Neurosci 2:55-58

20. Zubkov AY, Lewis AI, Raila FA, Zhang J, Parent AD (2000) Risk factors for the development of post-traumatic cerebral vasospasm. Surg Neurol 53:126-130

21. Zubkov AY, Pilkington AS, Bernanke DH, Parent AD, Zhang J (1999) Posttraumatic cerebral vasospasm: clinical and morphological presentations. J Neurotrauma 16:763-770 